\title{
Floquet dynamical quantum phase transitions in periodically quenched systems
}

\author{
Longwen Zhou ${ }^{1, \text { f }}$ and Qianqian $\mathrm{Du}^{1}$ \\ ${ }^{1}$ Department of Physics, College of Information Science and Engineering, \\ Ocean University of China, Qingdao, China 266100
}

(Dated: 2021-07-02)

\begin{abstract}
Dynamical quantum phase transitions (DQPTs) are characterized by nonanalytic behaviors of physical observables as functions of time. When a system is subject to time-periodic modulations, the nonanalytic signatures of its observables could recur periodically in time, leading to the phenomena of Floquet DQPTs. In this work, we systematically explore Floquet DQPTs in a class of periodically quenched one-dimensional system with chiral symmetry. By tuning the strength of quench, we find multiple Floquet DQPTs within a single driving period, with more DQPTs being observed when the system is initialized in Floquet states with larger topological invariants. Each Floquet DQPT is further accompanied by the quantized jump of a dynamical topological order parameter, whose values remain quantized in time if the underlying Floquet system is prepared in a gapped topological phase. The theory is demonstrated in a piecewise quenched lattice model, which possesses rich Floquet topological phases and is readily realizable in quantum simulators like the nitrogen-vacancy center in diamonds. Our discoveries thus open a new perspective for the Floquet engineering of DQPTs and the dynamical detection of topological phase transitions in Floquet systems.
\end{abstract}

\section{INTRODUCTION}

Dynamical quantum phase transitions are featured by the nonanalyticity of physical observables in time domain [1, 2]. They are usually triggered by a sudden [3-11] or a slow [12, 13] quench across an equilibrium quantum phase transition point, and will exhibit topological signatures when the pre- and post-quench systems are set in different topological phases 14 19. In the past few years, various aspects of DQPTs have been explored in theory (see [20 24] for reviews), and also demonstrated experimentally in a broad range of quantum simulator platforms 25] 35], yielding an intriguing perspective in the classification and characterization of nonequilibrium phases of matter.

Recently, the study of DQPTs has been extended to time-periodic driven systems [35 37], which possess rich nonequilibrium phases like the Floquet topological matter 38, 43, and time crystals 44 48. Following periodic driving fields, a different class of DQPT unique to Floquet systems has been discovered theoretically and also observed experimentally in a harmonically driven spin chain, which is thus named Floquet DQPTs [35. The nonanalytic cusps in the return rate of Floquet DQPTs are found to recur periodically in time with a non-decaying global profile, which is distinguished from the conventional DQPTs that are usually observable only in transient time scales [1. Moreover, when the periodically driven system is initialized in a Floquet topological phase, the Floquet DQPTs would also show topologically nontrivial signatures, characterized by the quantized jump of a dynamical topological order parameter (DTOP) at each critical time of the transition 35. In later studies, different aspects of Floquet DQPTs have

\footnotetext{
* zhoulw13@u.nus.edu
}

also been explored, such as the effect of mixed state and the property of entanglement spectrum [49, 50]. However, all the studies on Floquet DQPTs till now focus on a specific type of driving protocol, in which the system can be described by a static effective Hamiltonian in a rotating frame [35, 49, 50. Therefore, richer signatures of Floquet DQPTs under more general driving schemes have not been revealed. For example, is it possible to induce several Floquet DQPTs within a single driving period, making the nonanalytic cusps in the rate function of return probability to form a "sublattice structure in time"? Besides, are there any differences in DQPTs between systems initialized in Floquet phases with small and large topological invariants? Moreover, compared to the cases following a sudden or a slow quantum quench, much less about DQPTs is known when the quenches are applied over finite time durations [51, 52]. The resolution of these issues would further uncover the intrinsic properties of Floquet DQPTs, and establish their connections with Floquet topological matter and other nonequilibrium dynamical phenomena.

Motivated by these considerations, we explore Floquet DQPTs in a general class of periodically quenched lattice model, which possesses the chiral (sublattice) symmetry at each instant of time. In Sec. II], we introduce our theoretical framework, which shows that multiple Floquet DQPTs can indeed emerge within a single driving period under large quench amplitudes. Furthermore, a DTOP for Floquet DQPTs can be constructed from the noncyclic geometric phase of the evolving Floquet states [35. The DTOP shows a quantized jump whenever a Floquet DQPT happens, and remains to be an integer otherwise if the system is initially prepared in a gapped Floquet topological phase. Specially, the DTOP could take non-quantized values in certain time domains if the initial state of the system belongs to a gapless Floquet phase. One can therefore employ the DTOP to identify the boundaries between different Floquet topo- 
logical phases. In Sec. III, we demonstrate our theory in a prototypical and experimentally realizable periodically quenched lattice model, unveiling the rich signatures of Floquet DQPTs by detailed analytical and numerical calculations. In Sec. [V], we summarize our results and discuss potential future directions.

\section{THEORY}

In this section, we introduce our theory for the description of Floquet DQPTs in a typical class of periodically quenched system. Throughout our discussion, we will set the Planck constant $\hbar=1$ and the driving period $T=2$ in dimensionless units.

\section{A. Setup and Floquet dynamics}

In momentum presentation, the time-dependent Hamiltonian $H(k, t)$ of our periodically quenched lattice takes the form

$$
H(k, t)= \begin{cases}h_{x}(k) \sigma_{x} & t \in[2 \ell, 2 \ell+1) \\ h_{y}(k) \sigma_{y} & t \in[2 \ell+1,2 \ell+2) .\end{cases}
$$

Here $k \in[-\pi, \pi)$ is the quasimomentum defined in the first Brillouin zone. $\ell \in \mathbb{Z}$ counts the number of driving periods. $\sigma_{x, y, z}$ are Pauli matrices in their usual representations, and we will denote the $2 \times 2$ identity matrix as $\sigma_{0}$. It is clear that $H(k, t)$ is periodic in time with the period $T=2$, i.e., $H(k, t)=(k, t+2)$. We will also use the symbol $s$ to denote the time within a driving period, i.e., the micromotion time, so that any physical time $t$ can be decomposed as

$$
t=s+2 \ell, \quad \ell \in \mathbb{Z}, \quad s \in[0,2) .
$$

Meanwhile, according to Eq. (1), $H(k, t)$ has the chiral symmetry $\Gamma=\sigma_{z}$ at any instant of time, i.e., $\Gamma H(k, t) \Gamma=$ $-H(k, t)$ and $\Gamma^{2}=\sigma_{0}$. This allows one to characterize the topological phases of the system by integer winding numbers [53 55]. Note in passing that Eq. (1) represents one of the typical quench protocols for the realization of one-dimensional (1D) Floquet systems with chiral (sublattice) symmetry and nontrivial topological properties. The Pauli matrices in Eq. (1) can be replaced by any two of the three Pauli spins $\sigma_{x, y, z}$, and $h_{x, y}$ can be arbitrary functions of the quasimomentum $k$. Various other periodic kicking or quenching protocols for 1D chiral symmetric lattice models can be reduced to the form of Eq. (1), such as those reported in Refs. [53, 54]. Therefore, the theoretical framework developed here is expected to be appliable to Floquet DQPTs in a broad range of periodically quenched and continuously driven [35] chiral symmetric two-band systems.

We are interested in such a dynamical process, in which the system is initialized in a uniformly filled Floquet band and then undergoes nonequilibrium evolution governed by the piecewise quenched $H(k, t)$. The initial state of the system is obtained by solving the Floquet eigenvalue equation $U(k)|\psi(k)\rangle=e^{-i E(k)}|\psi(k)\rangle$, where $|\psi(k)\rangle$ is the Floquet eigenstate with eigenphase $E(k)$. The Floquet operator $U(k)$, referring to the evolution operator of the system over a complete driving period $T$ (e.g., from $t=$ $0^{-}$to $\left.t=0^{-}+2\right)$, takes the form

$$
U(k)=e^{-i h_{y}(k) \sigma_{y}} e^{-i h_{x}(k) \sigma_{x}},
$$

according to Eq. (1). Taking the Taylor expansion of the two terms on the right hand side of Eq. (3), we can express $U(k)$ equivalently as

$$
\begin{aligned}
U(k) & =\cos \left[h_{x}(k)\right] \cos \left[h_{y}(k)\right] \sigma_{0} \\
& -i \sin \left[h_{x}(k)\right] \cos \left[h_{y}(k)\right] \sigma_{x} \\
& -i \sin \left[h_{y}(k)\right] \cos \left[h_{x}(k)\right] \sigma_{y} \\
& +i \sin \left[h_{x}(k)\right] \sin \left[h_{y}(k)\right] \sigma_{z},
\end{aligned}
$$

which can be further recast into a single exponential form as

$$
U(k)=e^{-i E(k) \vec{n}(k) \cdot \vec{\sigma}}=e^{-i \vec{d}(k) \cdot \vec{\sigma}} .
$$

Here the eigenphase dispersion reads

$$
E(k)=\arccos \left\{\cos \left[h_{x}(k)\right] \cos \left[h_{y}(k)\right]\right\},
$$

$\vec{\sigma}=\left(\sigma_{x}, \sigma_{y}, \sigma_{z}\right)$, and the components of unit vector $\vec{n}=$ $\left[n_{x}(k), n_{y}(k), n_{z}(k)\right]$ are explicitly given by

$$
\begin{aligned}
& n_{x}(k)=\sin \left[h_{x}(k)\right] \cos \left[h_{y}(k)\right] / \sin [E(k)], \\
& n_{y}(k)=\sin \left[h_{y}(k)\right] \cos \left[h_{x}(k)\right] / \sin [E(k)], \\
& n_{z}(k)=-\sin \left[h_{x}(k)\right] \sin \left[h_{y}(k)\right] / \sin [E(k)] .
\end{aligned}
$$

We could then obtain the Floquet eigenstates by solving the eigenvalue equation of the effective Floquet Hamiltonian, i.e.,

$$
H_{\text {eff }}(k)\left|\psi_{ \pm}(k)\right\rangle=E_{ \pm}(k)\left|\psi_{ \pm}(k)\right\rangle,
$$

where the effective Hamiltonian $H_{\text {eff }}(k)$ and eigenphase bands $E_{ \pm}(k)$ are given by

$$
H_{\mathrm{eff}}(k)=\vec{d}(k) \cdot \vec{\sigma}, \quad E_{ \pm}(k)= \pm E(k),
$$

and the explicit expressions of Floquet eigenstates $\left|\psi_{ \pm}(k)\right\rangle$ are found to be

$$
\left|\psi_{v}(k)\right\rangle=\frac{1}{\sqrt{2 E_{v}(k)\left[E_{v}(k)-d_{z}(k)\right]}}\left[\begin{array}{c}
d_{x}(k)-i d_{y}(k) \\
E_{v}(k)-d_{z}(k)
\end{array}\right],
$$

where $v= \pm$ will be used to denote the signs of the eigenphase dispersions $E_{ \pm}(k)$. With these considerations, the time evolution operator of the system from time $t=0$ to a later time $t=s+2 \ell$ [see Eq. [2] ] can be written as 


$$
\begin{aligned}
& U(k, t)=U(k, s) U^{\ell}(k), \text { where } \\
& U(k, s)= \begin{cases}e^{-i s h_{x}(k) \sigma_{x}}, & s \in[0,1) \\
e^{i(2-s) h_{y}(k) \sigma_{y}} U(k) . & s \in[1,2)\end{cases}
\end{aligned}
$$

Here $U(k)$ is the Floquet operator of the system over a complete driving period, as given by Eq. (3) [56]. The evolution of a Floquet eigenstate $\left|\psi_{v}(k)\right\rangle(v= \pm)$ from $t=0$ to $t=s+2 \ell$ is then described by $\mid \psi_{v}(k, t=s+$ $2 \ell)\rangle=U(k, s) U^{\ell}(k)\left|\psi_{v}(k)\right\rangle$, which can be expressed more explicitly as

$$
\left|\psi_{v}(k, t)\right\rangle=e^{-i \ell E_{v}(k)} e^{-i s h_{x}(k) \sigma_{x}}\left|\psi_{v}(k)\right\rangle
$$

for $s \in[0,1)$ and

$$
\left|\psi_{v}(k, t)\right\rangle=e^{-i(\ell+1) E_{v}(k)} e^{i(2-s) h_{y}(k) \sigma_{y}}\left|\psi_{v}(k)\right\rangle
$$

for $s \in[1,2)$, respectively. Since $\left\{\left|\psi_{v}(k)\right\rangle \mid v= \pm\right\}$ in Eq. 10 form a complete and orthonormal basis, the dynamics of our periodically quenched system is completely solved by Eqs. (12)-13 once the explicit expressions of $h_{x}(k)$ and $h_{y}(k)$ are specified.

\section{B. Return amplitude and conditions of Floquet DQPTs}

With these preparations, we can now investigate the Floquet DQPTs in our class of piecewise quenched system. The central object in the study of DQPTs is the return amplitude (or return probability), which may be viewed as a dynamical version of the partition function [20]. For an evolution from time $t=0$ to $t=s+2 \ell$ [see Eq. (2)], the return amplitude of a single FloquetBloch state $\left|\psi_{v}(k)\right\rangle(v= \pm)$ is given by

$$
G_{v}(k, t)=\left\langle\psi_{v}(k)|U(k, t)| \psi_{v}(k)\right\rangle=e^{-i \ell E_{v}(k)} G_{v}(k, s),
$$

where

$$
G_{v}(k, s)=\left\langle\psi_{v}(k)|U(k, s)| \psi_{v}(k)\right\rangle
$$

describes the return amplitude of initial state $\left|\psi_{v}(k)\right\rangle$ at a given $k \in[-\pi, \pi)$ in micromotion time $s \in[0,2)$. Following the theory of Floquet DQPTs [35, if a state $\left|\psi_{v}(k)\right\rangle$ with $k=k_{c}$ happens to evolve to its orthogonal state at a critical time $s=s_{c}$, we will have $G_{v}\left(k_{c}, s_{c}\right)=0$, and the rate function of return probability (to be defined later) will become nonanalytic in the thermodynamic limit, signifying Floquet DQPTs at time $t_{c}=s_{c}+2 \ell$ for all $\ell \in \mathbb{Z}$.

The conditions of Floquet DQPTs are determined by the expressions of critical momentum $k_{c}$ and critical time $t_{c}$. For our periodically quenched system, the forms of $k_{c}$ and $t_{c}$ can be found analytically. Using Eqs. (12)(15) and the fact that $\left\langle\psi_{v}(k)\left|\sigma_{j}\right| \psi_{v}(k)\right\rangle=v n_{j}(k)$ for $j=$ $x, y, z$ [see Eq. (7) for $n_{x, y, z}(k)$ ], we find that

$G_{v}(k, s)=\left\{\begin{array}{l}\cos \left[s h_{x}(k)\right]-i \sin \left[s h_{x}(k)\right] v n_{x}(k), \\ e^{-i E_{v}(k)}\left\{\cos \left[s^{\prime} h_{y}(k)\right]+i \sin \left[s^{\prime} h_{y}(k)\right] v n_{y}(k)\right\}\end{array}\right.$

where $s \in[0,1), s^{\prime} \equiv 2-s \in[0,1)$ and $\left[n_{x}(k), n_{y}(k)\right]$ are the components of unit vector $\vec{n}(k)$ in Eq. (7). The expressions of $k_{c}$ and $s_{c}$ are then determined by the solutions of $G_{v}(k, s)=0$, yielding the following two sets of critical conditions

$$
\left\{\begin{array}{l}
s h_{x}(k)=\frac{2 p-1}{2} \pi \quad \& \quad \sin \left[h_{x}(k)\right] \cos \left[h_{y}(k)\right]=0 \\
s^{\prime} h_{y}(k)=\frac{2 q-1}{2} \pi \quad \& \quad \sin \left[h_{y}(k)\right] \cos \left[h_{x}(k)\right]=0
\end{array}\right.
$$

for $s \in[0,1)$ and $s^{\prime} \equiv 2-s \in[0,1)$. Here $p, q \in \mathbb{Z}$ and Eq. (7) has been used to reach the second equality in each set of condition. Note that these conditions do not depend on the values of $v(= \pm)$, implying that we would have the same Floquet DQPT conditions for the initial state being prepared in either Floquet band. By straightforward calculations, we see that in each half of the driving period, the condition in Eq. (17) generates two possible sets of solutions for the critical momenta and time. For $s \in[0,1)$, we have $G_{v}\left(k_{c}, s_{c}\right)=0$ if

$$
\begin{cases}h_{x}\left(k_{c}\right)=m \pi, & m \in \mathbb{Z} \neq 0 \\ s_{c}=\frac{2 p-1}{2 m} \in(0,1), & p \in \mathbb{Z}\end{cases}
$$

or

$$
\begin{cases}h_{y}\left(k_{c}\right)=\frac{2 m-1}{2} \pi, & m \in \mathbb{Z} \\ s_{c}=\frac{2 p-1}{2 h_{x}\left(k_{c}\right)} \in(0,1) . & p \in \mathbb{Z}\end{cases}
$$

For $s \in[1,2)$, we have $G_{v}\left(k_{c}, s_{c}\right)=0$ if

$$
\begin{cases}h_{y}\left(k_{c}\right)=n \pi, & n \in \mathbb{Z}_{\neq 0} \\ s_{c}^{\prime}=\frac{2 q-1}{2 n} \in(0,1), & q \in \mathbb{Z}\end{cases}
$$

or

$$
\begin{cases}h_{x}\left(k_{c}\right)=\frac{2 n-1}{2} \pi, & n \in \mathbb{Z} \\ s_{c}^{\prime}=\frac{2 q-1}{2 h_{y}\left(k_{c}\right)} \in(0,1), & q \in \mathbb{Z}\end{cases}
$$

where $s_{c}^{\prime}=2-s_{c}$. Given the explicit forms of $h_{x}(k)$ and $h_{y}(k)$, these conditions then determine all possible critical momenta $k_{c}$ and critical time $t_{c}=s_{c}+2 \ell(\ell \in \mathbb{Z})$ of Floquet DQPTs in the class of periodically quenched systems considered in this work. Note in passing that the critical time $s_{c}$ in Eqs. (18) and (20) take universal forms and independent of the explicit expressions of $h_{x}(k)$ and $h_{y}(k)$, which indicates their connections with the global nature of the underlying Floquet states. In the meantime, we notice that when the Floquet eigenphase spectrum of the system becomes gapless, i.e., when 
$E_{ \pm}(k)=0$ or $E_{ \pm}(k)= \pm \pi$ in Eq. (6), we would obtain

$$
\begin{cases}h_{x}\left(k_{0}\right)=m \pi, & m \in \mathbb{Z} \\ h_{y}\left(k_{0}\right)=n \pi, & n \in \mathbb{Z}\end{cases}
$$

where the set $\left\{k_{0}\right\}$ gives the locations at which the eigenphase band-gap closes in $k$-space, which is usually accompanied by a transition between different Floquet topological phases [54]. From Eqs. (18) and (20), it is clear that once $m, n \neq 0$ in Eq. (22), the gapless quasimomenta $\left\{k_{0}\right\}$ of the Floquet spectrum are also critical momenta of the Floquet DQPTs, yielding critical times at $s_{c}$ and $2-s_{c}$ within a driving period. Since the set $\left\{k_{0}\right\}$ is generally different for different values of $(m, n)$ in Eq. 22], the possible critical momenta and critical times in Eqs. (18) and 20) will also be different in general when the system is initialized in different Floquet topological phases. This allows one to discriminate Floquet topological phases from the behaviors of Floquet DQPTs, thus extending the connection between Floquet DQPTs and Floquet topological matter found in Ref. [35] to more general situations.

\section{Rate function of return probability}

We next introduce the rate function of return probability, which is the observable to show the time-domain nonanalyticity of Floquet DQPTs explicitly. Following Eq. (14), the return probability of a Floquet state $\left|\psi_{v}(k)\right\rangle$ $(v= \pm)$ evolved from time zero to $t$ is given by

$$
g_{v}(k, t)=\left|\left\langle\psi_{v}(k)|U(k, t)| \psi_{v}(k)\right\rangle\right|^{2}=g_{v}(k, s),
$$

where

$$
g_{v}(k, s)=\left|\left\langle\psi_{v}(k)|U(k, s)| \psi_{v}(k)\right\rangle\right|^{2},
$$

and $t=s+2 \ell$ for $s \in[0,2), \ell \in \mathbb{Z}$. It is clear that $g_{v}(k, t)$ possesses the same temporal periodicity as the system Hamiltonian, i.e., $g_{v}(k, t)=g_{v}(k, t+2)$. The complete behavior of $g_{v}(k, t)$ at any time $t$ is then determined once $g_{v}(k, s)$ is obtained. For our periodically quenched system, $g_{v}(k, s)$ takes the following explicit form according to Eq. 16

$g_{v}(k, s)= \begin{cases}\cos ^{2}\left[s h_{x}(k)\right]+\sin ^{2}\left[s h_{x}(k)\right] n_{x}^{2}(k), & s \in[0,1) \\ \cos ^{2}\left[s^{\prime} h_{y}(k)\right]+\sin ^{2}\left[s^{\prime} h_{y}(k)\right] n_{y}^{2}(k), & s \in[1,2)\end{cases}$

where $s^{\prime}=2-s \in[0,1)$. Again, we notice that $g_{v}(k, s)$ is independent of $v(= \pm)$, i.e., in which band the system is initialized, and $g_{v}(k, s)=0$ at each given set of critical momentum and time $(k, s)=\left(k_{c}, s_{c}\right)$ in Eqs. (18)-(21). The latter suggests us to consider the rate function of return probability $f_{v}(t)$, defined as

$$
f(t)=-\lim _{N \rightarrow \infty} \frac{1}{N} \sum_{k} \ln [g(k, t)]=-\int_{-\pi}^{\pi} \frac{d k}{2 \pi} \ln [g(k, t)],
$$

where $N$ is the number of degrees of freedom in the system (e.g., the number of unit cells), which is taken to be infinity in the thermodynamic limit. We have also denoted $g_{v}(k, s)$ by $g(k, s)$ in Eq. 26), as the former is independent of $v$. The rate function thus defined may then be viewed as a "free energy density" of the Floquet dynamics. Since $f(t)$ is a periodic function of time with the period $T=2$, i.e., $f(t)=f(t+2)$, we have for any time $t=s+2 \ell$ with $s \in[0,2)$ and $\ell \in \mathbb{Z}$ that

$$
f(t)=f(s)=-\int_{-\pi}^{\pi} \frac{d k}{2 \pi} \ln [g(k, s)]
$$

If $g(k, s)$ vanishes at certain critical time and momentum $\left(k_{c}, t_{c}\right), \ln \left[g\left(k_{c}, s_{c}\right)\right]$ will diverge, and after the integration over $k, f(s)$ could become nonanalytic at time $t_{c}=s_{c}+2 \ell$ for all $\ell \in \mathbb{Z}$, which are referred to as Floquet DQPTs. For 1D systems, a Floquet DQPT is usually manifested as a cusp in the profile of $f(t)$ around $t=t_{c}$ 35. Using Eqs. 25) and (27), we can further obtain the explicit expression of the return rate function $f(s)$ for our periodically quenched system, i.e.,

$$
f(s)=-\int_{-\pi}^{\pi} \frac{d k}{2 \pi} \cdot\left\{\begin{array}{l}
\ln \left\{\cos ^{2}\left[s h_{x}(k)\right]+\sin ^{2}\left[s h_{x}(k)\right] n_{x}^{2}(k)\right\} \\
\ln \left\{\cos ^{2}\left[s^{\prime} h_{y}(k)\right]+\sin ^{2}\left[s^{\prime} h_{y}(k)\right] n_{y}^{2}(k)\right\}
\end{array}\right.
$$

for $s \in[0,1)$ and $s^{\prime}=2-s \in[0,1)$, respectively. The profile of $f(s)$ over a driving period $s \in[0,2)$ then contains all the information about the time-domain nonanalytic properties of Floquet DQPTs.

\section{Geometric phase and DTOP}

In previous studies, dynamical topological order parameters (DTOPs) have been introduced to characterize DQPTs following a sudden quench [14, 15], or Floquet DQPTs induced by harmonic driving fields [35]. The values of a DTOP, obtained from the winding number of the noncyclic (Pancharatnam) geometric phase of the evolving state over the first Brillouin zone at each time, usually show a quantized jump at every critical time of a DQPT or Floquet DQPT, and remain quantized otherwise. It thus plays the role of a topological order parameter of the dynamics. In this work, we extend the DTOP-characterization of Floquet DQPTs to our periodically quenched setting.

The noncyclic geometric phase is given by the difference between the total phase and dynamical phase of the return amplitude $G_{v}(k, t)(v= \pm)$. Following Eq. (15), 
the total phase is

$$
\phi_{v}(k, t)=-i \ln \left[\frac{G_{v}(k, t)}{\left|G_{v}(k, t)\right|}\right]=-\ell E_{v}(k)+\phi_{v}(k, s),
$$

where we have set $t=s+2 \ell$ for $s \in[0,2)$ and $\ell \in \mathbb{Z}$ to reach the second equality. Using Eq. (16), we obtain the micromotion part of the total phase $\phi_{v}(k, s)$ for our periodically quenched setting as

$$
\phi_{v}(k, s)=\left\{\begin{array}{l}
-\arctan \left\{\tan \left[s h_{x}(k)\right] v n_{x}(k)\right\}, \\
\arctan \left\{\tan \left[s^{\prime} h_{y}(k)\right] v n_{y}(k)\right\}-E_{v}(k),
\end{array}\right.
$$

for $s \in[0,1)$ and $s^{\prime}=2-s \in[0,1)$, respectively. The dynamical phase of the return amplitude is defined as

$$
\phi_{v}^{\mathrm{D}}(k, t)=-\int_{0}^{t} d t^{\prime}\left\langle\psi_{v}\left(k, t^{\prime}\right)\left|H\left(k, t^{\prime}\right)\right| \psi_{v}\left(k, t^{\prime}\right)\right\rangle .
$$

Using the time-periodicity of Hamiltonian $H(k, t)$ and the direct result of Floquet theorem $\left|\psi_{v}(k, t+2)\right\rangle=$ $e^{-i E_{v}(k)}\left|\psi_{v}(k, t)\right\rangle, \phi_{v}^{\mathrm{D}}(k, t)$ can be further expressed as

$$
\phi_{v}^{\mathrm{D}}(k, t)=\ell \phi_{v}^{\mathrm{D}}(k, 2)+\phi_{v}^{\mathrm{D}}(k, s),
$$

where we have set $t=s+2 \ell$ with $s \in[0,2)$ and $\ell \in \mathbb{Z}$ on the right hand side of the equality. For our periodically quenched system, we find with the help of Eqs. (1), 110, 12 and 13 that $\phi_{v}^{\mathrm{D}}(k, 2)=-v \vec{h}(k) \cdot \vec{n}(k)$ and

$$
\phi_{v}^{\mathrm{D}}(k, s)= \begin{cases}-v s h_{x}(k) n_{x}(k), & s \in[0,1) \\ -v\left[\vec{h}(k) \cdot \vec{n}(k)-s^{\prime} h_{y}(k) n_{y}(k)\right], & s \in[1,2)\end{cases}
$$

where $s^{\prime}=2-s$. Note that the dynamical phase is closely related to the relative angle between the components $\left[h_{x}(k), h_{y}(k), 0\right]$ of the time-dependent Hamiltonian $H(k, t)$ and the components $\left[n_{x}(k), n_{y}(k), n_{z}(k)\right]$ of the Floquet effective Hamiltonian in each half of the driving period. Taking the difference between the total phase and dynamical phase, we find the noncyclic geometric phase [35] to be

$$
\phi_{v}^{\mathrm{G}}(k, t)=\phi_{v}(k, t)-\phi_{v}^{\mathrm{D}}(k, t),
$$

whose explicit expression for our periodically quenched system can be obtained directly from Eqs. (29)-(33). At a fixed time $t$, the winding number of $\phi_{v}^{\mathrm{G}}(k, t)$ in $k$-space then determines the DTOP $w_{v}(t)$, given by

$$
w_{v}(t)=\int \frac{d k}{2 \pi} \partial_{k} \phi_{v}^{\mathrm{G}}(k, t) .
$$

When the evolution of the system passes a critical time $t_{c}$ of the Floquet DQPTs, the value of $w_{v}(t)$ is expected to show a quantized jump [35], i.e.,

$$
w_{v}\left(t_{c}^{+}\right)-w_{v}\left(t_{c}^{-}\right) \in \mathbb{Z},
$$

where the exact amount of jump depends on the configuration of the critical momenta set $\left\{k_{c}\right\}$ in the first Brillouin zone. For certain specific models, the integration range of $k$ in Eq. (36) could be chosen as a subset of the first Brillouin zone $k \in[-\pi, \pi)$ due to the possible translation and reflection symmetries of $\phi_{v}^{\mathrm{G}}(k, t)$ in $k$-space. For example, if we have $h_{j}(k \pm \pi)=-h_{j}(k)$ for $j=x, y$, both $\phi_{v}(k, t)$ and $\phi_{v}^{\mathrm{D}}(k, t)$ will be invariant under the translation over $\pi$ in $k$-space, and the same is true for $\phi_{v}^{\mathrm{G}}(k, t)$. So the range of integration in Eq. (36) can be confined to $k \in[0, \pi]$ in this case. More examples about the reduction of integration range in the calculation of $w_{v}(t)$ will be made clear in the next section.

To sum up, we have established a theoretical framework to analyze Floquet DQPTs in a typical class of periodically quenched lattice model. The critical conditions as summarized in Eqs. (18)-(21) clearly suggest that by enlarging the amplitudes of quench functions $h_{x}(k)$ and $h_{y}(k)$, the exact number of Floquet DQPTs that could occur in each driving periodic can be put under flexible control. This highlights one of the key advantages of Floquet engineering in the realization of DQPTs. Besides, we have observed the connection between Floquet DQPTs and the underlying Floquet topological phases of the periodically quenched system, and also introduced a DTOP to characterize the topological signatures of Floquet DQPTs. In the following section, we will demonstrate our results with a prototypical periodically quenched lattice model, which is rich in Floquet DQPTs and experimentally realizable at the same time.

\section{RESULTS}

In this section, we demonstrate our theoretical scheme of Floquet DQPTs in a piecewise quenched lattice (PQL) model, which possesses rich Floquet topological phases and also being realizable in quantum simulator setups.

\section{A. The PQL model}

Our explicit model is obtained by setting

$$
h_{x}(k)=J_{x} \cos k, \quad h_{y}(k)=J_{y} \sin k,
$$

in the general Hamiltonian Eq. (1), leading to the Floquet operator

$$
U(k)=e^{-i J_{y} \sin k \sigma_{y}} e^{-i J_{x} \cos k \sigma_{x}} .
$$

Here the parameters $J_{x}$ and $J_{y}$ control the amplitudes of the two quenches within each half of the driving period. Physically, they can be interpreted as the kicking strengths of a spin-1/2 quantum kicked rotor [54], or the hopping amplitudes of a particle on a tight-binding lattice [57. In the former case, the system may be realized by the setup proposed in Refs. [58, 59, which achieves the 
quantum walk in momentum space by ${ }^{87} \mathrm{Rb}$ BEC atoms. In the latter case, the system may be realized by replacing the harmonic driving fields with square-wave pulses in the NV center experimental setup of Refs. [35, 60]. Theoretically, rich Floquet topological phases have been identified in this model, which are characterized by larger topological invariants and multiple topological edge modes [54]. This further motivates us to choose this model as our working example to demonstrate the key features of Floquet DQPTs in periodically quenched systems.

From now on, we take the quench amplitudes $J_{x, y}>0$ without loss of generality. Using Eqs. (37) and (38), the Floquet operator can be written in a single exponential form as $U(k)=e^{-i E(k) \vec{n}(k) \cdot \vec{\sigma}}$, where $\cos [E(k)]=$ $\cos \left(J_{x} \cos k\right) \cos \left(J_{y} \sin k\right)$ and the unit vector $\vec{n}(k)=$ $\left[n_{x}(k), n_{y}(k), n_{z}(k)\right]$, with

$$
\begin{aligned}
& n_{x}(k)=\frac{\sin \left(J_{x} \cos k\right) \cos \left(J_{y} \sin k\right)}{\sin [E(k)]}, \\
& n_{y}(k)=\frac{\sin \left(J_{y} \sin k\right) \cos \left(J_{x} \cos k\right)}{\sin [E(k)]} \\
& n_{z}(k)=-\frac{\sin \left(J_{x} \cos k\right) \sin \left(J_{y} \sin k\right)}{\sin [E(k)]} .
\end{aligned}
$$

The Floquet effective Hamiltonian then takes the form $H_{\text {eff }}=E(k) \vec{n}(k) \cdot \vec{\sigma}=\vec{d}(k) \cdot \vec{\sigma}$, with the corresponding eigenvalue equation $H_{\text {eff }}(k)\left|\psi_{ \pm}(k)\right\rangle=E_{ \pm}(k)\left|\psi_{ \pm}(k)\right\rangle$, whose explicit solutions are given by Eq. 10p. Following Eq. (22), the gapless quasimomenta of the model satisfy $J_{x} \cos k_{0}=m \pi$ and $J_{y} \sin k_{0}=n \pi$ for $m, n \in \mathbb{Z}$, yielding the curves that describing the boundaries between different Floquet topological phases, i.e., $m^{2} \pi^{2} / J_{x}^{2}+$ $n^{2} \pi^{2} / J_{y}^{2}=1$ for $|m| \leq J_{x} / \pi$ and $|n| \leq J_{y} / \pi$ [54]. According to the discussion in the last section, the solutions of $k_{0}$ also generate a set of critical momenta $k_{c}$ for the Floquet DQPTs when $m, n \neq 0$.

Using Eqs. (18)-21], we could obtain the solutions of critical momenta and critical times for the PQL model if the system is initialized in a Floquet eigenstate. In the first half of a driving period, i.e., for $s \in[0,1)$, the set of $\left(k_{c}, s_{c}\right)$ satisfy

$$
\begin{cases}k_{c}=\arccos \left(\frac{m \pi}{J_{x}}\right), & m \in \mathbb{Z}_{\neq 0} \cap|m| \leq \frac{J_{x}}{\pi} \\ s_{c}=\frac{2 p-1}{2 m} \in[0,1), & p \in \mathbb{Z}\end{cases}
$$

or

$$
\begin{cases}k_{c}=\arcsin \left(\frac{2 m-1}{2 J_{y}} \pi\right), & m \in \mathbb{Z} \cap\left|m-\frac{1}{2}\right| \leq \frac{J_{y}}{\pi} \\ s_{c}=\frac{2 p-1}{2 J_{x} \cos k_{c}} \pi \in[0,1) . & p \in \mathbb{Z}\end{cases}
$$

Similarly, in the second half of a driving period, i.e., for $s \in[1,2)$, the set $\left(k_{c}, s_{c}=2-s_{c}^{\prime}\right)$ satisfy

$$
\begin{cases}k_{c}=\arcsin \left(\frac{n \pi}{J_{y}}\right), & n \in \mathbb{Z}_{\neq 0} \cap|n| \leq \frac{J_{y}}{\pi} \\ s_{c}^{\prime}=\frac{2 q-1}{2 n} \in[0,1), & q \in \mathbb{Z}\end{cases}
$$

or

$$
\begin{cases}k_{c}=\arccos \left(\frac{2 n-1}{2 J_{x}} \pi\right), & n \in \mathbb{Z} \cap\left|n-\frac{1}{2}\right| \leq \frac{J_{x}}{\pi} \\ s_{c}^{\prime}=\frac{2 q-1}{2 J_{y} \sin k_{c}} \pi \in[0,1) . & q \in \mathbb{Z}\end{cases}
$$

Eqs. (40)-(43) provide us with analytical solutions for all possible critical momenta $k_{c}$ and critical times $t_{c}=s_{c}+2 \ell$ $(\ell \in \mathbb{Z})$ of the Floquet DQPTs in the PQL model. The notable connection between the expressions of $k_{c}$ in Eqs. (40), 420 and the gapless quasimomentum $k_{0}$ satisfying $k_{0}=\arccos \left(m \pi / J_{x}\right)=\arcsin \left(n \pi / J_{y}\right)$ for $m, n \in \mathbb{Z}$ also allows one to obtain qualitatively different patterns of Floquet DQPTs in different Floquet topological phases of the PQL model, as will be demonstrated explicitly by numerical calculations.

\section{B. Numerical results}

In the following, we present typical examples of Floquet DQPTs in the PQL model by evaluating the rate function of return probability in Eq. (26), noncyclic geometric phase in Eq. (34) and DTOP in Eq. (35). We first evolve the system according to Eq. 23) for each Floquet eigenstate, and then obtain the return rate from Eq. (26) by numerically evaluating the integral over quasimomentum $k$. The geometric phase is obtained by taking the difference between the total phase and the dynamical phase of the return amplitude following Eqs. (29) and (31). After numerically obtaining the geometric phase, we compute the DTOP in terms of Eq. (35). In the computation of the integral, $N=300$ grids in $k$-space is taken, which is enough for the convergence of the numerical data in order to reproduce the nonanalytic signals of Floquet DQPTs in the thermodynamic limit (corresponding to $N \rightarrow \infty)$. Note that for the PQL model, we have $h_{j}(k \pm \pi)=-h_{j}(k)$ for $j=x, y$ according to Eq. (37), implying that the geometric phase has a translational symmetry over $\pi$ in $k$-space. Furthermore, since $h_{x}(k)\left[h_{y}(k)\right]$ and $n_{x}(k)\left[n_{y}(k)\right]$ are even (odd) functions of $k$, the geometric phase also has a reflection symmetry with respect to $k=0$ according to Eqs. (30), 33) and (34). Therefore, in the calculation of DTOP in Eq. (35) for the PQL model, we could restrict the range of integration over $k$ to one quarter of the first Brillouin zone, e.g., $k \in[0, \pi / 2]$. As a further simplification, we notice that the order of the two quenches are swapped under the combined effects of parameter exchange $J_{x} \leftrightarrow J_{y}$ and momentum shift $k \rightarrow \pi / 2-k$. The result of such a swap is simply a shifting of the Floquet DQPTs from one half of the driving period to the other half, as directly deducible from Eq. (28). Therefore, for $J \neq J^{\prime}$, the Floquet DQPTs in the system with $\left(J_{x}, J_{y}\right)=\left(J, J^{\prime}\right)$ and $\left(J_{x}, J_{y}\right)=\left(J^{\prime}, J\right)$ can be directly related with each other. For $J=J^{\prime}$, each Floquet DQPT happens at the micromotion time $s_{c}$ has a mirror that happens at $2-s_{c}$ for all $t_{c}=s_{c}+2 \ell$ with $\ell \in \mathbb{Z}$.

In the first set of results, we consider the initial state of 

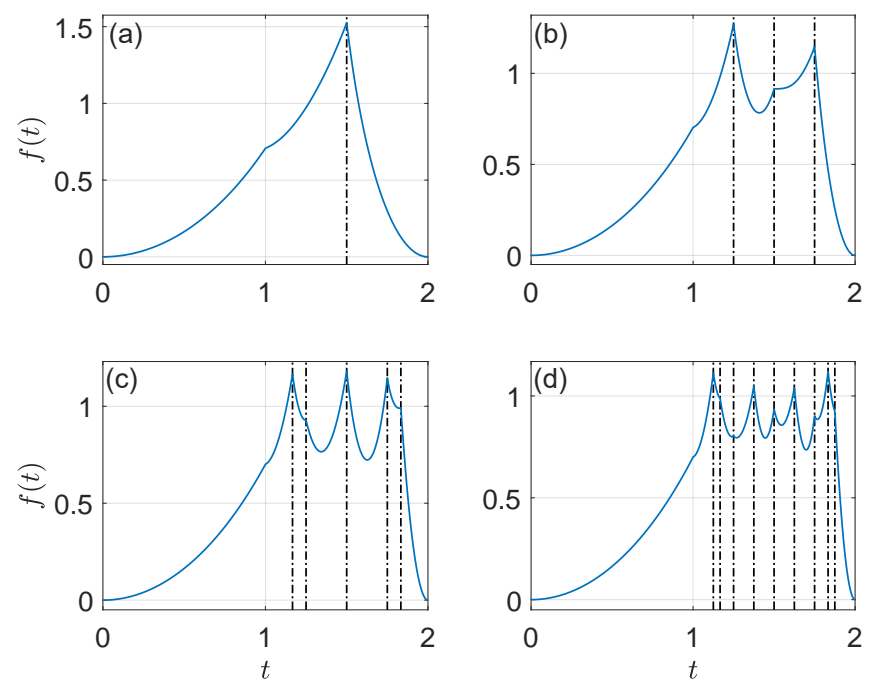

FIG. 1. Rate function $f(t)$ of the PQL model (blue solid lines) over a driving period $t \in[0,2]$, in which a Floquet DQPT manifests as a cusp at the corresponding critical time. System parameters are set as $J_{x}=0.5 \pi$ for all panels and $J_{y}=1.1 \pi, 2.1 \pi, 3.1 \pi, 4.1 \pi$ for panels (a), (b), (c) and (d), respectively. The crossing points of black dash-dotted lines with the horizontal axis in each panel refer to the critical times $t_{c}$ of Floquet DQPTs in each case, which are obtained analytically from Eq. 42 .
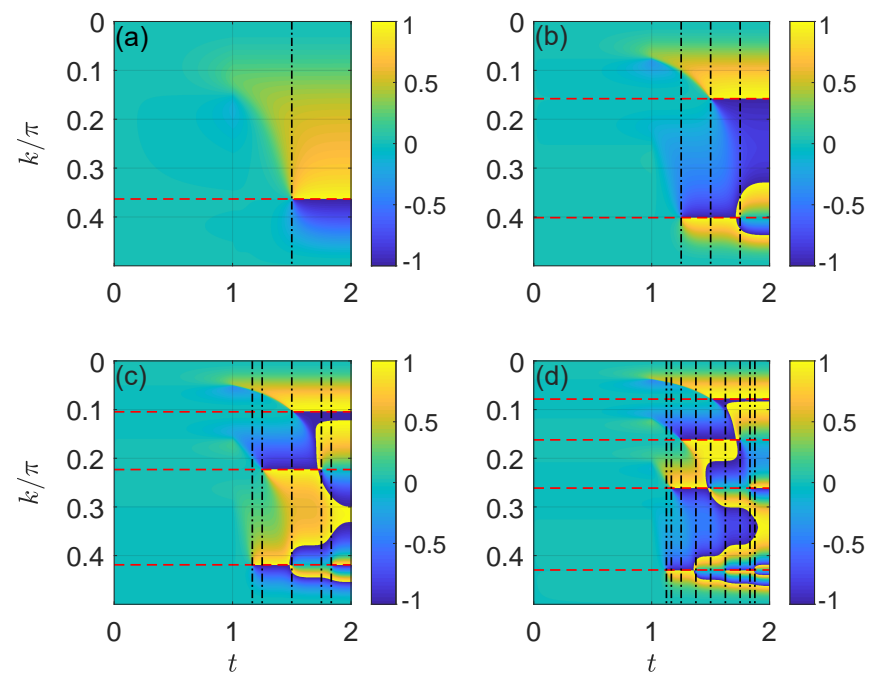

FIG. 2. Noncyclic geometric phase $\phi_{-}^{\mathrm{G}}(k, t) / \pi$ of the PQL model versus the quasimomentum $k \in[0, \pi / 2]$ and time $t \in$ $[0,2]$. System parameters are set as $J_{x}=0.5 \pi$ for all panels and $J_{y}=1.1 \pi, 2.1 \pi, 3.1 \pi, 4.1 \pi$ for panels (a), (b), (c) and (d), respectively. In each panel, the crossing points of the horizontal red dashed lines with the vertical axis correspond to the critical momenta $k_{c}$, and the intersection points of the vertical black dash-dotted lines with the horizontal axis refer to the critical times $t_{c}$, which are obtained from Eq. 42, analytically for each chosen set of system parameters.
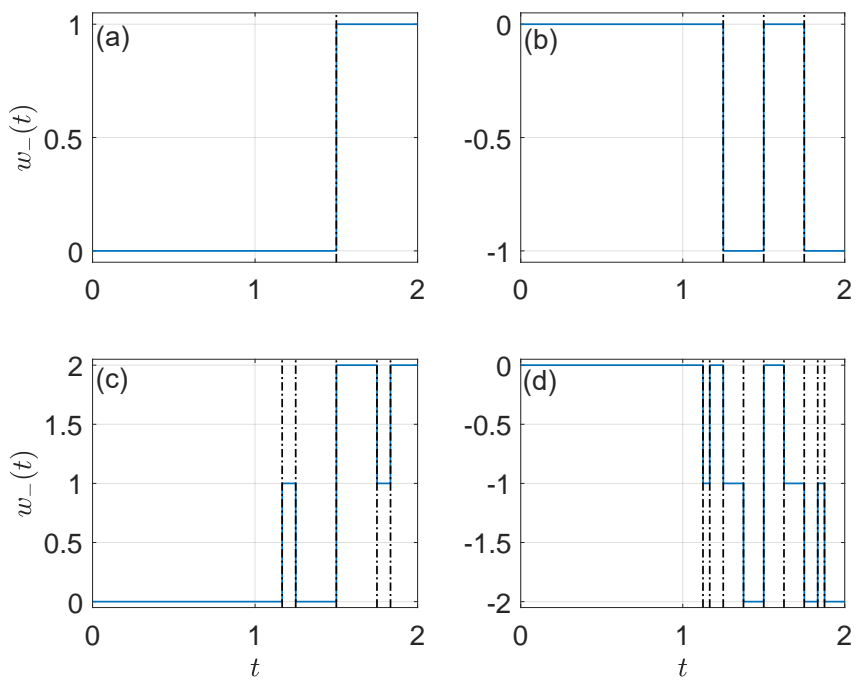

FIG. 3. DTOP $w_{-}(t)$ of the PQL model (blue solid lines) versus time over a driving period $t \in[0,2]$. System parameters are chosen to be $J_{x}=0.5 \pi$ for all panels and $J_{y}=1.1 \pi, 2.1 \pi, 3.1 \pi, 4.1 \pi$ for panels (a), (b), (c) and (d). In each panel, the crossing points of the vertical black dashdotted lines with the horizontal axis refer to the critical times $t_{c}$, which are obtained analytically with the chosen system parameters from Eq. 42. The DTOP makes a quantized jump at each critical time $t_{c}$ and remaining quantized otherwise.

the PQL model to be prepared in the Floquet band with quasienergy $E_{-}(k)$ at each quasimomentum $k \in[-\pi, \pi)$, and present the rate function of return probability $f(t)$ [Eq. [28], noncyclic geometric phase $\phi_{-}^{\mathrm{G}}(k, t)$ [Eq. [34] and DTOP $w_{-}(t)$ [Eq. [35)] versus time over a driving period $t=0 \rightarrow 2$ in Figs. 1, 2 and 3, respectively. The system parameters are set as $J_{x}=0.5 \pi$ for all figure panels and $J_{y}=1.1 \pi, 2.1 \pi, 3.1 \pi, 4.1 \pi$ in the panels (a), (b), (c) and (d) of each figure, respectively. According to Ref. [54, the PQL model with these four sets of system parameters belong to four different Floquet topological phases, with larger topological invariants at larger values of $J_{y}$. In Fig. 1 , we observe that with the increase of $J_{y}$, the system undergoes more and more Floquet DQPTs within a single driving period, with a cusp observed at every critical time $t_{c}$ of the DQPT. The possible values of critical times are predicted precisely by the critical condition in Eq. (42), whose solutions of $k_{c}$ are closely related to the gapless quasimomenta $k_{0}$ of the system. Specially, more Floquet DQPTs are observed when the initial phase of the PQL model carries larger topological invariants (see the phase diagram in Fig. 1 of Ref. [54]). This implies the possibility of discriminating different Floquet topological phases of the PQL model from Floquet DQPTs, and also introduces a route to engineer more Floquet DQPTs in a finite time window with the help of Floquet topological phases. Besides, the global profiles of $f(t)$ in Fig. 1 do not decay with time thanks to the applied driving field, which allow us to observe and detect the nonanalytic signatures of Floquet DQPTs in 

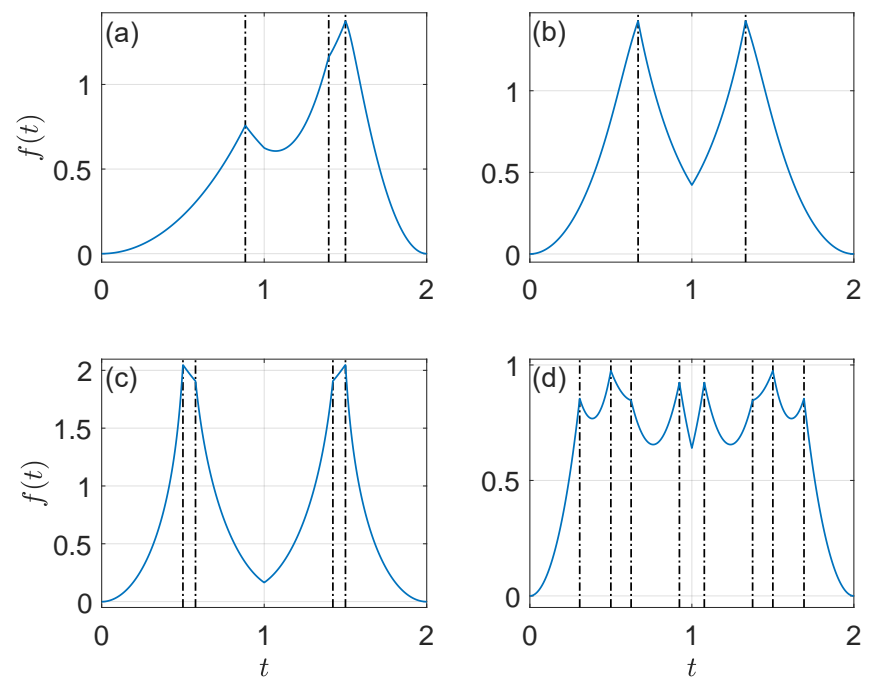

FIG. 4. Rate function $f(t)$ of the PQL model (blue solid lines) versus time over a driving period, in which a Floquet DQPT manifests as a cusp at the corresponding critical time. System parameters are set as $\left(J_{x}, J_{y}\right)=(0.6 \pi, 1.5 \pi)$ for panel (a), $\left(J_{x}, J_{y}\right)=(0.9 \pi, 0.9 \pi)$ for panel $(\mathrm{b}),\left(J_{x}, J_{y}\right)=(\pi, \pi)$ for panel $(\mathrm{c})$, and $\left(J_{x}, J_{y}\right)=(1.7 \pi, 1.7 \pi)$ for panel $(\mathrm{d})$. The intersection points of black dash-dotted lines with the horizontal axis in each panel give the critical times $t_{c}$ of Floquet DQPTs in each case, which are obtained analytically from Eqs. 40-43.

a larger time window compared with the case in conventional DQPTs following a single quench [20]. The noncyclic geometric phase $\phi_{-}^{\mathrm{G}}(k, t)$, as presented in Fig. 2 also possesses a $2 \pi$-jump around the critical momenta $k_{c}$ (red dashed lines) at each critical time $t_{c}$ (black dash-dotted lines), reflecting the discontinuity in the time-derivatives of the rate function $f(t)$ at the Floquet DQPTs. After the integration over $k \in[0, \pi / 2]$, the $2 \pi$-change of $\phi_{-}^{\mathrm{G}}(k, t)$ (corresponding to the sudden change of colors from dark blue to dark yellow or the opposite in each figure panel) finally leads to the integer quantized jump of DTOP at each critical time (black dash-dotted lines) of the Floquet DQPT, as presented in Fig. 3. Except at the critical times, the DTOP remain quantized over other time durations, and thus playing the role of a topological order parameter for Floquet DQPTs. Notably, in Figs. 3(c) and 3(d), $w_{-}(t)$ possesses a change $\Delta w_{-}=2$ at $t_{c}=1.5$. Such a jump of DTOP in a step bigger than 1 is not observed in DQPTs following a single quantum quench, and may thus be a unique feature of DQPTs following time-periodic drivings or other more complicated nonequilibrium protocols.

In the second set of results, we still prepare the initial state of the system in the Floquet band of the PQL model with negative eigenphases at all $k \in[-\pi, \pi)$, and investigate the Floquet DQPTs following quenches over a complete driving period. The quench amplitudes are chosen as $\left(J_{x}, J_{y}\right)=(0.6 \pi, 1.5 \pi),(0.9 \pi, 0.9 \pi),(\pi, \pi)$ and $(1.7 \pi, 1.7 \pi)$ for the results presented in panels $(\mathrm{a}),(\mathrm{b})$,
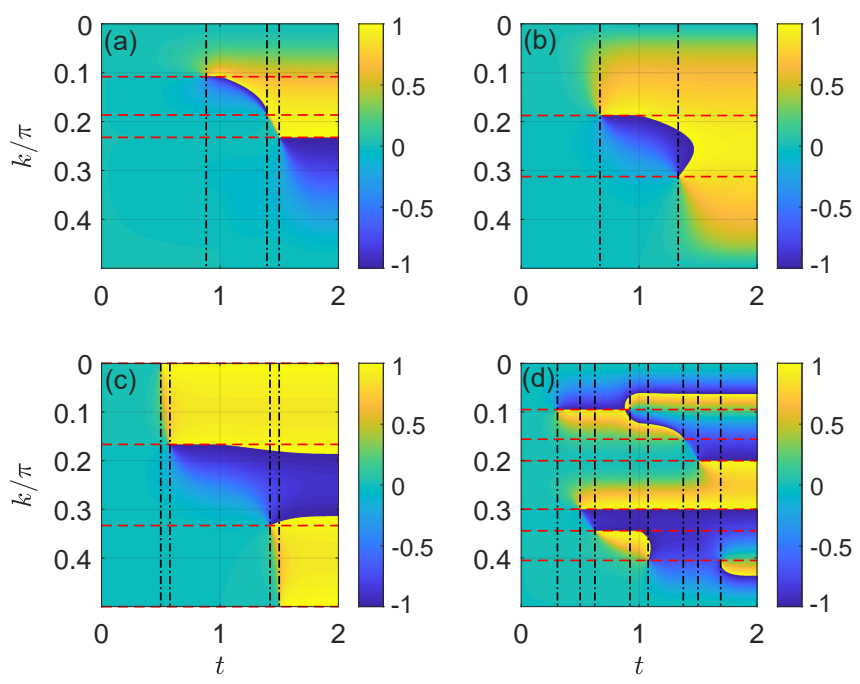

FIG. 5. Noncyclic geometric phase $\phi_{-}^{\mathrm{G}}(k, t) / \pi$ of the PQL model with respect to the quasimomentum $k \in[0, \pi / 2]$ and time $t \in[0,2]$. System parameters are set as $\left(J_{x}, J_{y}\right)=$ $(0.6 \pi, 1.5 \pi)$ for panel (a), $\left(J_{x}, J_{y}\right)=(0.9 \pi, 0.9 \pi)$ for panel (b), $\left(J_{x}, J_{y}\right)=(\pi, \pi)$ for panel $(\mathrm{c})$, and $\left(J_{x}, J_{y}\right)=(1.7 \pi, 1.7 \pi)$ for panel (d). In each panel, the intersection points of the horizontal red dashed lines with the vertical axis refer to the critical momenta $k_{c}$, and the crossing points of the vertical black dash-dotted lines with the horizontal axis correspond to the critical times $t_{c}$, both of which are obtained from Eqs. 40 (43) analytically for each chosen set of system parameters.
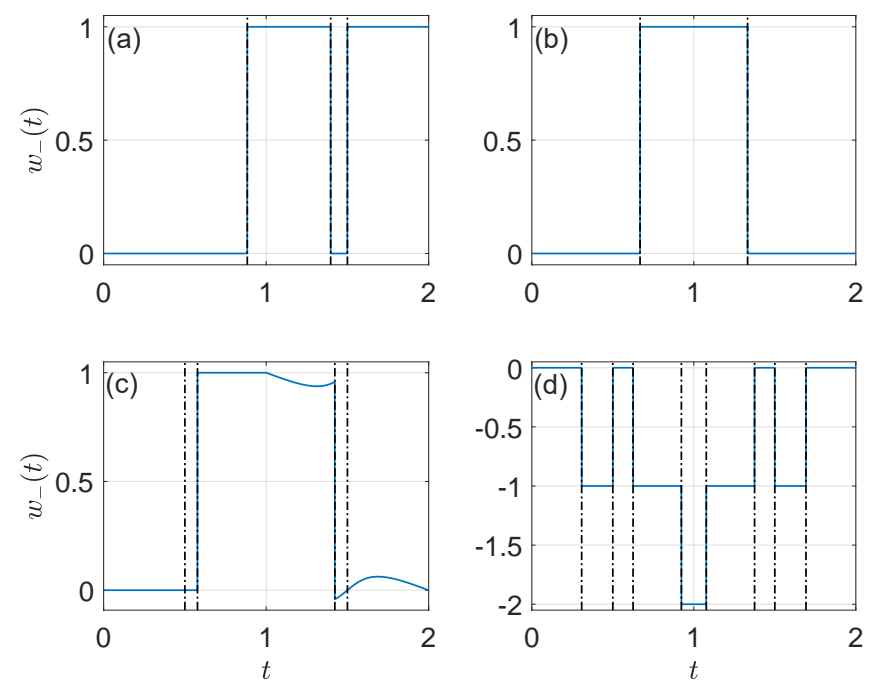

FIG. 6. DTOP $w_{-}(t)$ of the PQL model (blue solid lines) versus time over a driving period $t \in[0,2]$. System parameters are set as $\left(J_{x}, J_{y}\right)=(0.6 \pi, 1.5 \pi)$ for panel (a), $\left(J_{x}, J_{y}\right)=(0.9 \pi, 0.9 \pi)$ for panel $(\mathrm{b}),\left(J_{x}, J_{y}\right)=(\pi, \pi)$ for panel $(\mathrm{c})$, and $\left(J_{x}, J_{y}\right)=(1.7 \pi, 1.7 \pi)$ for panel $(\mathrm{d})$. In each panel, the intersection points of the vertical black dash-dotted lines with the horizontal axis correspond to the critical times $t_{c}$, which are obtained analytically from Eqs. (40)- 43 with the chosen system parameters. The DTOP makes a quantized jump at each $t_{c}$ except in panel (c), where the system is initialized in a gapless Floquet phase. 
(c) and (d), respectively, of the Figs. 4, 5 and 6 In panels (a)-(d) of Fig. 4. we observe Floquet DQPTs within each half of the driving period, with the critical times $t_{c}$ (black dash-dotted lines) determined analytically by Eqs. (40)- (43). Notably, when $J_{x}=J_{y}$, we found the critical times $t_{c}$ to be symmetric with respect to $t=T / 2=1$, which is originated from the symmetry of the rate function $f(t)$ under the exchange of quenching amplitudes $J_{x}$ and $J_{y}$, as discussed before in this subsection. Besides, we also observe the general tendency of having more Floquet DQPTs under larger quenching amplitudes, which again highlights the flexibility of the Floquet approach in the engineering and control of DQPTs within a finite time domain. In Fig. 5, we observe that the noncyclic geometric phase $\phi_{-}^{\mathrm{G}}(k, t)$ develops a $2 \pi$-jump around $k=k_{c}$ (red dashed lines) every time when the system evolves across a critical time $t_{c}$ (black dash-dotted lines) in each figure panel (corresponding to the sudden change of colors from dark blue to dark yellow or the opposite), which provide geometric signatures of the nonanalyticity for Floquet DQPTs. Finally, in Fig. 6, we present the DTOP $w_{-}(t)$ versus time over a driving period, as obtained from Eq. (35). In Figs. 6(a), (b) and (d), the PQL system is initialized in different Floquet topological phases [54, and the qualitatively different behaviors of $w_{-}(t)$ again allow us to distinguish different Floquet topological phases with the help of Floquet DQPTs. Interestingly, we observe anomalous behaviors in the DTOP in Fig. 6(c), in the sense that it either changes smoothly in certain time windows (but still with quantized jumps at the critical times), or shows no signatures of jump across certain critical times. The underlying reason behind such an anomaly is that for the case considered in Fig. 66(c), the system is initialized in a gapless Floquet phase, instead of a gapped "ground state" usually employed in the study of DQPTs. Therefore, the Floquet DQPTs could also provide us with a useful dynamical tool to locate the phase boundaries between different Floquet topological matter. For completeness, we have also explored the Floquet DQPTs in other parameter domains of the PQL model, and obtained results that are consistent with the general principles as presented in this section, thus verifying the theoretical framework introduced in Sec. II of this work. As a final comment, for initial states prepared in topologically trivial Floquet phases, no signatures of Floquet DQPTs have been reported in the previous study [35]. However, this does not exclude the possibility of observing Floquet DQPTs in systems prepared at trivial initial states, as also notified before in the study of non-Floquet DQPTs following a single quench [15. Therefore, the most general relationship between the topological nature of Floquet states and Floquet DQPTs deserves more investigations, which will be left for future studies.

\section{SUMMARY}

In this work, we introduced a framework to describe Floquet DQPTs in periodically quenched 1D systems with chiral symmetry. Within a driving period, we observed a series of Floquet DQPTs, which are characterized by cusps in the rate function of return probability with a non-decaying global profile, and quantized jumps of the dynamical topological order parameter (DTOP). These nonanalytic features all repeat periodically in time with the same period as the driving field. Via tuning the strengths of quenches, both the critical momenta, critical times and number of Floquet DQPTs within a driving period can be put under flexible control, revealing the advantage of Floquet engineering in the realization of DQPTs. Furthermore, the DTOP behaves differently when the underlying Floquet system is prepared in a gapped topological phase or in a gapless phase, and can thus be used as an efficient probe to the topological phase transitions in Floquet systems. Our theoretical scheme is further verified in a prototypical piecewise quenched lattice model, which holds rich Floquet topological phases and phase transitions [54].

By changing the driving field realized in Ref. [35] from the harmonic form to the square-wave form, we expect our predicted signatures of Floquet DQPTs to be readily detectable in the setup containing an NV center in diamond [35, 60. In the experiment reported in Ref. [35], the finite coherence time of electron spin and the imperfection of microwave pulses may cause uncertainties. The nearly vanishing return probabilities around the critical points of Floquet DQPTs could also introduce some errors in the measurement of rate function at the critical times. However, the main features of Floquet DQPTs in connection with cusps in the rate function and jumps in the DTOP could still be clearly seen over a time window of three driving periods $(6 \mu \mathrm{s})$, as shown in Ref. [35]. Such a time window is long enough for one to observe Floquet DQPTs in periodically quenched systems, and the lifetime of Floquet states is also comparable to the observed time of Floquet DQPTs in the experiment [35]. Very recently, the periodically quenched lattice model introduced in Eq. (38) have been simulated experimentally, and its Floquet topological phases have also been observed in Ref. 61] with an NV center setup by measuring the time-averaged spin textures. Specially, the coupling strengths $\left(J_{x}, J_{y}\right)$ can reach $3 \pi$ in dimensionless units in the experiment of Ref. [61], which is enough for one to observe the high values of DTOP reported in Fig. 3 It is clear that the same experimental setting can also be used to detect the Floquet DQPTs predicted in this work.

Putting together, our discoveries uncover the nonanalytic, geometric and topological nature of Floquet DQPTs in periodically quenched systems, which not only go beyond the conventional studies of DQPTs in suddenquench and slow-quench settings, but also emphasize the power of Floquet engineering in controlling DQPTs and the usefulness of DQPTs in detecting topological phases 
and phase transitions in Floquet systems. In future work, it would be interesting to extend our framework to Floquet systems with more complicated driving protocols, non-Hermitian Floquet systems [3, 62, 69], multiband systems [70, many-particle Floquet systems with disorder and many-body interactions, and checking the existence and robustness of Floquet DQPTs in these complicated settings.

\section{ACKNOWLEDGEMENT}

L.Z. is supported by the National Natural Science Foundation of China (Grant No. 11905211), the China Postdoctoral Science Foundation (Grant No. 2019M662444), the Fundamental Research Funds for the Central Universities (Grant No. 841912009), the Young Talents Project at Ocean University of China (Grant No. 861801013196), and the Applied Research Project of Postdoctoral Fellows in Qingdao (Grant No. 861905040009).
[1] M. Heyl, A. Polkovnikov, and S. Kehrein, Dynamical Quantum Phase Transitions in the Transverse-Field Ising Model, Phys. Rev. Lett. 110, 135704 (2013).

[2] F. Pollmann, S. Mukerjee, A. G. Green, and J. E. Moore, Dynamics after a sweep through a quantum critical point, Phys. Rev. E 81, 020101(R) (2010).

[3] L. Zhou, Q. Wang, H. Wang, and J. Gong, Dynamical quantum phase transitions in non-Hermitian lattices, Phys. Rev. A 98, 022129 (2018).

[4] M. Heyl, Dynamical Quantum Phase Transitions in Systems with Broken-Symmetry Phases, Phys. Rev. Lett. 113, 205701 (2014).

[5] E. Canovi, P. Werner, and M. Eckstein, First-Order Dynamical Phase Transitions, Phys. Rev. Lett. 113, 265702 (2014).

[6] S. Vajna and B. Dóra, Disentangling dynamical phase transitions from equilibrium phase transitions, Phys. Rev. B 89, 161105(R) (2014).

[7] M. Heyl, Scaling and Universality at Dynamical Quantum Phase Transitions, Phys. Rev. Lett. 115, 140602 (2015).

[8] Z. Huang and A. V. Balatsky, Dynamical Quantum Phase Transitions: Role of Topological Nodes in Wave Function Overlaps, Phys. Rev. Lett. 117, 086802 (2016).

[9] M. Heyl, Quenching a quantum critical state by the order parameter: Dynamical quantum phase transitions and quantum speed limits, Phys. Rev. B 95, 060504(R) (2017).

[10] R. Jafari, H. Johannesson, A. Langari, and M. A. MartinDelgado, Quench dynamics and zero-energy modes: The case of the Creutz model, Phys. Rev. B 99, 054302 (2019).

[11] R. Jafari, Dynamical Quantum Phase Transition and Quasi Particle Excitation, Scientific Reports 9, 2871 (2019).

[12] S. Sharma, U. Divakaran, A. Polkovnikov, and A. Dutta, Slow quenches in a quantum Ising chain: Dynamical phase transitions and topology, Phys. Rev. B 93, 144306 (2016).

[13] U. Bhattacharya and A. Dutta, Interconnections between equilibrium topology and dynamical quantum phase transitions in a linearly ramped Haldane model, Phys. Rev. B 95, 184307 (2017).

[14] S. Vajna and B. Dóra, Topological classification of dynamical phase transitions, Phys. Rev. B 91, 155127 (2015).
[15] J. C. Budich and M. Heyl, Dynamical topological order parameters far from equilibrium, Phys. Rev. B 93, 085416 (2016).

[16] C. Yang, L. Li, and S. Chen, Dynamical topological invariant after a quantum quench, Phys. Rev. B 97, 060304(R) (2018).

[17] L. Pastori, S. Barbarino, and J. C. Budich, Signatures of topology in quantum quench dynamics and their interrelation, Phys. Rev. Research 2, 033259 (2020).

[18] Z. Gong and M. Ueda, Topological EntanglementSpectrum Crossing in Quench Dynamics, Phys. Rev. Lett. 121, 250601 (2018).

[19] N. Sedlmayr, P. Jaeger, M. Maiti, and J. Sirker, Bulkboundary correspondence for dynamical phase transitions in one-dimensional topological insulators and superconductors, Phys. Rev. B 97, 064304 (2018).

[20] M. Heyl, Dynamical quantum phase transitions: A review, Rep. Prog. Phys. 81, 054001 (2018).

[21] A. A. Zvyagin, Dynamical quantum phase transitions (Review Article), Low Temp. Phys. 42, 971 (2016).

[22] M. Heyl, Dynamical quantum phase transitions: A brief survey, EPL 125, 26001 (2019).

[23] Y. Chao and C. Shu, Topological invariant in quench dynamics, Acta Physica Sinica 68, 220304 (2019).

[24] T.-S. Deng and W. Yi, Fixed points and dynamic topological phenomena in quench dynamics, Acta Physica Sinica 68, 040303 (2019).

[25] P. Jurcevic, H. Shen, P. Hauke, C. Maier, T. Brydges, C. Hempel, B. P. Lanyon, M. Heyl, R. Blatt, and C. F. Roos, Direct Observation of Dynamical Quantum Phase Transitions in an Interacting Many-Body System, Phys. Rev. Lett. 119, 080501 (2017).

[26] J. Zhang, G. Pagano, P. W. Hess, A. Kyprianidis, P. Becker, H. Kaplan, A. V. Gorshkov, Z.-X. Gong, and C. Monroe, Observation of a many-body dynamical phase transition with a 53-qubit quantum simulator, Nature (London) 551, 601 (2017).

[27] N. Fläschner, D. Vogel, M. Tarnowski, B. S. Rem, D.-S. Lühmann, M. Heyl, J. C. Budich, L. Mathey, K. Sengstock, and C. Weitenberg, Observation of dynamical vortices after quenches in a system with topology, Nat. Phys. 14, 265 (2018).

[28] S. Smale, P. He, B. A. Olsen, K. G. Jackson, H. Sharum, S. Trotzky, J. Marino, A. M. Rey, and J. H. Thywissen, Observation of a transition between dynamical phases in a quantum degenerate Fermi gas, Sci. Adv. 5, eaax1568 (2018). 
[29] X. Guo, C. Yang, Y. Zeng, Y. Peng, H. Li, H. Deng, Y. Jin, S. Chen, D. Zheng, and H. Fan, Observation of Dynamical Quantum Phase Transition by a Superconducting Qubit Simulation, Phys. Rev. Appl. 11, 044080 (2019).

[30] T. Tian, Y. Ke, L. Zhang, S. Lin, Z. Shi, P. Huang, C. Lee, and J. Du, Observation of dynamical phase transitions in a topological nanomechanical system, Phys. Rev. B 100, 024310 (2019).

[31] K. Wang, X. Qiu, L. Xiao, X. Zhan, Z. Bian, W. Yi, and P. Xue, Simulating Dynamic Quantum Phase Transitions in Photonic Quantum Walks, Phys. Rev. Lett. 122, 020501 (2019).

[32] X.-Y. Xu, Q.-Q. Wang, M. Heyl, J. C. Budich, W.-W. Pan, Z. Chen, M. Jan, K. Sun, J.-S. Xu, Y.-J. Han, C.-F. Li, and G.-C. Guo, Measuring a Dynamical Topological Order Parameter in Quantum Walks, Light: Science \& Applications 9, 7 (2020).

[33] H.-X. Yang, T. Tian, Y.-B. Yang, L.-Y. Qiu, H.-Y. Liang, A.-J. Chu, C. B. Dağ, Y. Xu, Y. Liu, and L.-M. Duan, Observation of dynamical quantum phase transitions in a spinor condensate, Phys. Rev. A 100, 013622 (2019).

[34] T. Tian, H.-X. Yang, L.-Y. Qiu, H.-Y. Liang, Y.-B. Yang, Y. Xu, and L.-M. Duan, Observation of Dynamical Quantum Phase Transitions with Correspondence in an Excited State Phase Diagram, Phys. Rev. Lett. 124, 043001 (2020).

[35] K. Yang, L. Zhou, W. Ma, X. Kong, P. Wang, X. Qin, X. Rong, Y. Wang, F. Shi, J. Gong, and J. Du, Floquet dynamical quantum phase transitions, Phys. Rev. B 100, 085308 (2019).

[36] A. Kosior and K. Sacha, Dynamical quantum phase transitions in discrete time crystals, Phys. Rev. A 97, 053621 (2018).

[37] A. Kosior, A. Syrwid, and K. Sacha, Dynamical quantum phase transitions in systems with broken continuous time and space translation symmetries, Phys. Rev. A 98, 023612 (2018).

[38] J. Cayssol, B. Dóra, F. Simon, and R. Moessner, Floquet topological insulators, Phys. Status Solidi RRL 7, 101 (2013).

[39] M. S. Rudner and N. H. Lindner, Floquet topological insulators: from band structure engineering to novel nonequilibrium quantum phenomena, Nat. Rev. Phys. 2, 229 (2020).

[40] T. Oka and S. Kitamura, Floquet Engineering of Quantum Materials, Annu. Rev. Condens. Matter Phys. 10, 387 (2019).

[41] F. Harper, R. Roy, M. S. Rudner, S. L. Sondhi, Topology and Broken Symmetry in Floquet Systems, Annu. Rev. Condens. Matter Phys. 11, 345 (2020).

[42] L. Zhou, H. Wang, D. Y. H. Ho and J. Gong, Aspects of floquet bands and topological phase transitions in a continuously driven superlattice, Eur. Phys. J. B 87, 204 (2014).

[43] D. Y. H. Ho and J. Gong, Quantized Adiabatic Transport in Momentum Space, Phys. Rev. Lett. 109, 010601 (2012).

[44] K. Sacha and J. Zakrzewski, Time crystals: A review, Rep. Prog. Phys. 81, 016401 (2018).

[45] N. Y. Yao and C. Nayak, Time crystals in periodically driven systems, Phys. Today 71(9), 40 (2018).

[46] D. V. Else, C. Monroe, C. Nayak, and N. Y. Yao, Discrete Time Crystals, Annu. Rev. Condens. Matter Phys. 11,
467 (2020).

[47] L. Guo and P. Liang, Condensed matter physics in time crystals, New J. Phys. 22075003 (2020).

[48] V. Khemani, R. Moessner, S. L. Sondhi, A Brief History of Time Crystals, arXiv:1910.10745.

[49] S. Zamani, R. Jafari, and A. Langari, Floquet dynamical quantum phase transition in the extended XY model: Nonadiabatic to adiabatic topological transition, Phys. Rev. B 102, 144306 (2020).

[50] R. Jafari and A. Akbari, Floquet dynamical phase transition and entanglement spectrum, Phys. Rev. A 103, 012204 (2021).

[51] T. Puškarov and D. Schuricht, Time evolution during and after finite-time quantum quenches in the transverse-field Ising chain, SciPost Phys. 1, 003 (2016).

[52] A. A. Zvyagin, Fermionic quantum spin liquids: Exact results for parametric pumping, Phys. Rev. B 100, 024413 (2019).

[53] J. K. Asbóth and H. Obuse, Bulk-boundary correspondence for chiral symmetric quantum walks, Phys. Rev. B 88, 121406(R) (2013).

[54] L. Zhou and J. Gong, Floquet topological phases in a spin-1/2 double kicked rotor, Phys. Rev. A 97, 063603 (2018).

[55] L. Zhou and Q. Du, Floquet topological phases with fourfold-degenerate edge modes in a driven spin- $1 / 2$ Creutz ladder, Phys. Rev. A 101, 033607 (2020).

[56] Note that with the help of Eq. (3), the second line of Eq. 11 can be equivalently written as $U(k, s)=$ $e^{-i(s-1) h_{y}(k) \sigma_{y}} e^{-i h_{x}(k) \sigma_{x}}$ for $s \in[1,2)$. It describes the evolution of the system over the second half of a driving period.

[57] L. Zhou, Dynamical characterization of non-Hermitian Floquet topological phases in one dimension, Phys. Rev. B 100, 184314 (2019).

[58] G. Summy and S. Wimberger, Quantum random walk of a Bose-Einstein condensate in momentum space, Phys. Rev. A 93, 023638 (2016).

[59] S. Dadras, A. Gresch, C. Groiseau, S. Wimberger, and G. S. Summy, Quantum Walk in Momentum Space with a Bose-Einstein Condensate, Phys. Rev. Lett. 121, 070402 (2018).

[60] W. Ma, L. Zhou, Q. Zhang, M. Li, C. Cheng, J. Geng, X. Rong, F. Shi, J. Gong, and J. Du, Experimental Observation of a Generalized Thouless Pump with a Single Spin, Phys. Rev. Lett. 120, 120501 (2018).

[61] B. Chen, S. Li, X. Hou, F. Zhou, P. Qian, F. Mei, S. Jia, N. Xu, and H. Shen, Digital quantum simulation of Floquet topological phases with a solid-state quantum simulator, Photon. Res. 9, 81-87 (2021).

[62] L. Zhou and J. Gong, Non-Hermitian Floquet topological phases with arbitrarily many real-quasienergy edge states, Phys. Rev. B 98, 205417 (2018).

[63] L. Zhou and J. Pan, Non-Hermitian Floquet topological phases in the double-kicked rotor, Phys. Rev. A 100, 053608 (2019).

[64] L. Zhou, Non-Hermitian Floquet topological superconductors with multiple Majorana edge modes, Phys. Rev. B 101, 014306 (2020).

[65] J. Pan and L. Zhou, Non-Hermitian Floquet second order topological insulators in periodically quenched lattices, Phys. Rev. B 102, 094305 (2020). 
[66] L. Zhou, Non-Hermitian Floquet Phases with EvenInteger Topological Invariants in a Periodically Quenched Two-Leg Ladder, Entropy 22, 746 (2020).

[67] W. Zhao, L. Zhou, J. Liu, P. Tong, and K. Huang, Superexponential diffusion in nonlinear non-Hermitian systems, Phys. Rev. A 102, 062213 (2020).

[68] L. Zhou, Y. Gu, and J. Gong, Dual topological characterization of non-Hermitian Floquet phases, Phys. Rev.
B 103, L041404 (2021).

[69] L. Zhou and Q. Du, Non-Hermitian topological phases and dynamical quantum phase transitions: a generic connection, New J. Phys. in press, https://doi.org/10.1088/1367-2630/ac0574.

[70] T. Masłowski and N. Sedlmayr, Quasiperiodic dynamical quantum phase transitions in multiband topological insulators and connections with entanglement entropy and fidelity susceptibility, Phys. Rev. B 101, 014301 (2020). 